\title{
Review of Toxico-Kinetics of Nanogold through In Vivo and In Vitro Studies
}

\author{
Kavitha Palaniappan ${ }^{1}$ \\ ${ }^{1}$ Newcastle Australia Institute of Higher Education (a wholly owned entity of The University of Newcastle, Australia) \\ 100 Victoria Street, \#13-01/02, National Library Board Building, Singapore 188064 \\ Kavitha.Palaniappan@newcastle.edu.au
}

\begin{abstract}
Nanogold is one of the most explored nanomaterials, especially in the field of biomedicine. Due to its unique optical properties, it has found its way through various therapies, including the treatment of rheumatoid arthritis and a range of different types of cancers. Apart from its use in biomedicine, it is also used in environmental remediation, ranging from being used for oxidation of carbon monoxide as a catalyst and for the purification of hydrogen and water. Once it is utilized for its destined purpose, it may remain in the living body or enter the living body through the environment. Hence, it is essential to understand the biosafety of nanogold, especially in terms of its toxico-kinetics, so as to enable the policy makers to arrive at suitable standards to regulate the use of nanogold. This paper aims to review the toxico-kinetics, that is the mode of entry, biodistribution, bioaccumulation and potential toxicity of nanogold through the various in vivo and in vitro studies in the existing literature.
\end{abstract}

Keywords: Toxico-kinetics, Nanogold, Biomedicine, In vivo, In vitro.

\section{Introduction}

Nanotechnology is a field that is rapidly developing with uses in almost every field of study, ranging from medical therapies to environmental remediation. The rapid development of nanotechnology has also paved way for the need for another field which is nanotoxicology. Nanotechnology is a double-edged sword with loads of benefits on one side and potential toxic effects, which are yet to be studied and confirmed on the other side. Nanotechnology basically involves the use of substances that have less than 100nm in at least one dimension. Chemical substances behave in a completely different way, in terms of their physical and chemical properties when their size is reduced to the nanoscale. Gold is a typical example: in the bulk form, it is inert and hardly participates in any reaction; however, nanogold is a highly reactive substance and is also established and validated for its efficiency in treatment of rheumatoid arthritis [1].

Nanogold possesses several properties that favour its extensive usage, and their significant properties are shown in Fig. 1. The extremely small tunable sized nanogold can be synthesized in different forms such as rods, tubes, spheres, wires, stars, hexapods, etc [2]. Apart from the ease in modifying their surface structure and charges, several functional groups as per the requirement of the processes can also be easily added to nanogold making them more versatile in their usage [3]. Nanogold are also said to possess strong optical properties [4], surface plasmon resonance [5] and luminescence capability. Due to these properties, they are extensively used in the biomedical field for treatment purposes (Fig. 2), it becomes essential to study their toxicity, both in vitro and in vivo so that substantial standards for their usage in the biomedical field can be regulated. This paper is aiming to review the factors associated to the toxicity of nanogold, differences in toxicity based on the mode of entry into the living system, accumulation and distribution of nanogold in the system followed by in vivo and in vitro toxicity of nanogold. 


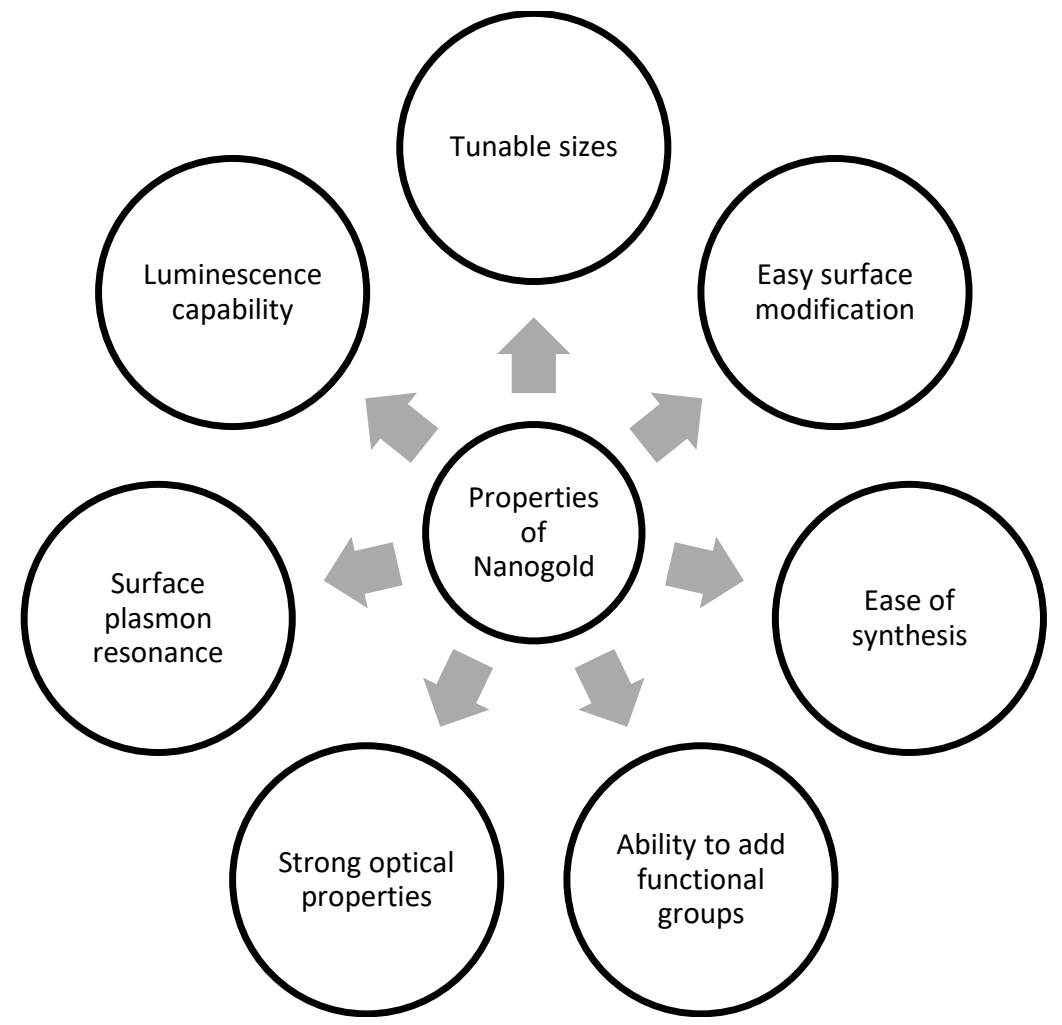

Fig. 1: Properties of nanogold that facilitate their use in the biomedical field

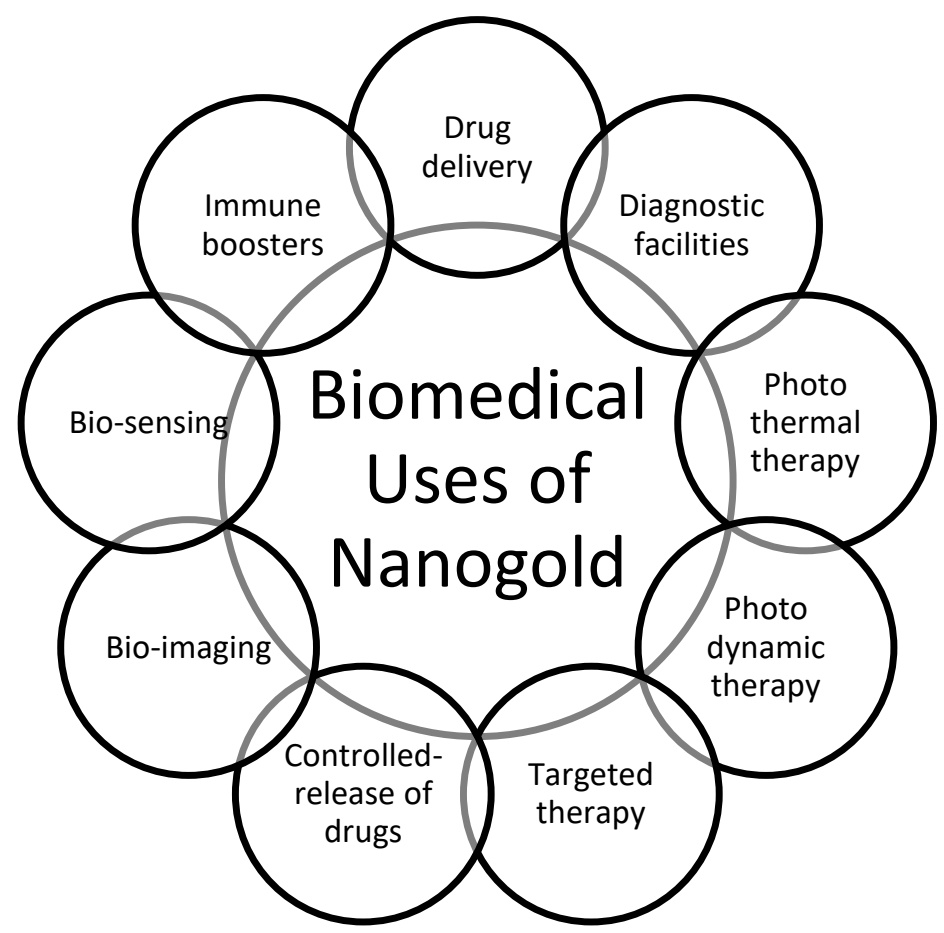

Fig. 2: Use of Nanogold in the Biomedical field 


\section{Factors Associated to the Toxicity of Nanogold}

Even though there are several properties of nanogold, the chief factors that have been identified in the literature to be associated to their toxicity are their size, shape, surface volume ratio and surface modification.

\subsection{Size of Nanogold}

The size of the nanomaterial basically decides the ability of the nanomaterial to cross potential cellular barriers within a living system and would also have a significant influence on the endocytosis capability of the material. The smaller the size of the nanomaterial, the easier it would be able to cross cellular barriers and can localize or accumulate within various cells and/or cellular organelles [6]. Different studies have shown different toxicity levels for a range of nanogold sizes and studies have also indicated that the toxicity is found to be on the higher side when the size of nanogold is smaller and the surface charge is negative [7]. This study also found that the accumulation of autophagosome is also influenced by the size of the nanogold particles [7]. Nanogold particles in the size range of around 10nm were the ones that were found to be widely distributed in all the organs [8].

\subsection{Shape and Surface Volume Ratio of Nanogold}

Compared to all the different shapes, nanogold spheres were found to be absorbed much more efficiently than their counter-shapes and they also showed more toxicity. Nanogold hexapods were found to be the least toxic among the different shapes of nanogold experimented so far in the literature. However, the cellular update of nanogold hexapods were considerably at a faster rate in vitro [9]. With respect to the surface volume ratio, the larger surface volume ratio was found to be more toxic, given that they provide an increased area for surface activity [10].

\subsection{Surface Modification of Nanogold}

Nanogold, by itself is considered to be toxic and hence surface modification is usually done to reduce the toxicity of nanogold. Further to that, modification is also done to attach different functional groups to the nanogold so that it can be used for the various reasons as highlighted above in the biomedical field. Cethyltrimethylammonium bromide (CTAB) is a commonly used agent that is added to maintain the rod shape of nanogold and CTAB has been attributed to cellular toxicity [11]. Another stabilizer that is also commonly used is sodium citrate and this was also found to be toxic in excess amounts when they were conjugated with nanogold [12].

\section{Toxico-kinetics of Nanogold}

Toxico-kinetics of any substance involves the mode of entry of the substance into the living system, followed by its distribution and accumulation within the system. Even though there are several in vitro studies done to demonstrate the toxicity of nanogold, the effects may vary significantly within a living system due to the various processes that the substance has to handle once inside a living body. Hence, the need arises to study the movement of substances within the body, that is, in vivo, in order to get a better understanding of the toxicity of the substances.

Toxico-kinetics begins with the entry of nanogold into the living system which can be through various means such as intraperitoneal, intravenous, dermal, inhalation, oral or subcutaneous. Once they come in contact with the biological units such as proteins, absorption of nanogold begins. Once absorbed, they can move around the body and accumulate at any convenient location or get metabolized or get modified or react with other substances within the living body or can also be eliminated [13].

\subsection{In vitro Toxicity of Nanogold}

In vitro studies are the ones that are usually performed at the start of toxicity studies as they are easy to perform and does not have the risk of harming any life. However, the results may not be fully applicable to a living unit as the predictions may not be accurate [14]. When MRC-5 human lung fibroblasts were subjected to nanogold, the genes and proteins associated to stress response were expressed followed by high lipid peroxidation [15]. In the same study, autophagy and oxidative stress was also noted in MRC-5 cells [15]. The viability of human colorectal adenocarcinoma cells (HT29) was 
significantly reduced in another study when exposed to nanogold [16]. Nanogold also increased the reactive oxygen species (ROS) and induced cytotoxic effects in human leukemia (HL-60) and hepatoma (HepG2) cell lines [17]. Citratecapped gold nanoparticles induced toxicity to the human carcinoma lung cells and the same study also noted that nanogold induced changes to the nuclear structure and cytotoxicity in A549 cells [18]. Even though toxicity at cell line levels have been established by various studies as explained above, nanogold did not increase the concentration of inflammatory markers and hence may not actually initiate an inflammatory response [19]. Some studies have also shown DNA damage caused by nanogold as small as $8 \mathrm{~nm}$ [20] and 20nm [21] respectively. Nanogold was found to have greater affinity towards thiol and amine groups [22] and this leads to the formation of free radicals, which in turn makes it more toxic [23].

\subsection{In vivo Toxicity of Nanogold}

In vivo studies with respect to nanogold have been done in animals such as rats and mice. Changes to gene expression [24] and DNA damage [25] was observed in rats. As far as accumulation is concerned, citrate-coated nanogold tended to accumulate in liver, spleen, neurons, kidney and was also found to cross the blood brain barrier in Wistar rats [26]. On the other hand, PEG-coated nanogold accumulated in the spleen and liver in rats in another study [27]. PEG-coated nanogold also was found to induce cytotoxicity due to the formation of reactive oxygen species (ROS) in rats [28], whereas nanogold caused mild changes to the kidney and liver sections in rats [29]. Inflammation of liver tissue was observed with in vivo tests performed on mice [30]. Weight loss and possibly a loss of appetite was noticed in mice due to naked colloidal nanogold [31]. In broiler chicken, nanogold caused significant toxicity that included histopathological changes, fragmentation of DNA, reduction in antibody titre against avian influenza and newcastle disease and even fragmentation of DNA [32].

\section{Conclusion}

Nanogold has extensive use in the biomedical field and researchers are constantly exploring the further use of it for various kinds of therapies. However, it is of utmost importance that we establish the biosafety parameters of nanogold, especially in terms of its toxicity once it enters the living body and its ability to bioaccumulate which can lead to more changes or issues much later before they can be commercially utilized for the different treatments. There are more and more in vivo studies going on in this field and time-to-time reviews that update the researchers on the current status of such studies is essential to keep them abreast of the recent findings.

\section{References}

[1] Y. Liu, F. Cao, B. Sun, J. A. Bellanti, and S. G. Zheng, "Magnetic nanoparticles: A new diagnostic and treatment platform for rheumatoid arthritis," J Leukoc Biol, vol. 109, no. 2, pp. 415-424, Feb. 2021, doi: 10.1002/JLB.5MR0420008RR.

[2] L. Vigderman and E. R. Zubarev, "High-Yield Synthesis of Gold Nanorods with Longitudinal SPR Peak Greater than $1200 \mathrm{~nm}$ Using Hydroquinone as a Reducing Agent," Chem. Mater., vol. 25, no. 8, pp. 1450-1457, Apr. 2013, doi: $10.1021 / \mathrm{cm} 303661 \mathrm{~d}$.

[3] C. Fernández-López, L. Polavarapu, M. D. Solis, M. J. Taboada, F. Obelleiro, R. Contreras-Caceres, I. Pastoriza-Santos, and J. Perez-Juste, "Gold Nanorod-pNIPAM Hybrids with Reversible Plasmon Coupling: Synthesis, Modeling, and SERS Properties,” ACS Appl. Mater. Interfaces, vol. 7, no. 23, pp. 12530-12538, Jun. 2015, doi: 10.1021/am5087209.

[4] Y. Zhang, L.-M. Wang, E.-Z. Tan, S.-H. Yang, L.-D. Li, and L. Guo, "Uniform arrays of gold nanoparticles with different surface roughness for surface enhanced Raman scattering," Chinese Chemical Letters, vol. 26, no. 11, pp. 1426-1430, Nov. 2015, doi: 10.1016/j.cclet.2015.06.004.

[5] X. Ke, S. Sarina, J. Zhao, X. Zhang, J. Chang, and H. Zhu, "Tuning the reduction power of supported gold nanoparticle photocatalysts for selective reductions by manipulating the wavelength of visible light irradiation," Chem. Commun., vol. 48, no. 29, p. 3509, 2012, doi: 10.1039/c2cc17977f. 
[6] S. Ahn, E. Seo, K. H. Kim, and S. J. Lee, "Physical Property Control on the Cellular Uptake Pathway and Spatial Distribution of Nanoparticles in Cells," j biomed nanotechnol, vol. 11, no. 6, pp. 1051-1070, Jun. 2015, doi: 10.1166/jbn.2015.2037.

[7] H. Yen, S. Hsu, and C. Tsai, "Cytotoxicity and Immunological Response of Gold and Silver Nanoparticles of Different Sizes," Small, vol. 5, no. 13, pp. 1553-1561, Jul. 2009, doi: 10.1002/smll.200900126.

[8] W. H. De Jong, W. I. Hagens, P. Krystek, M. C. Burger, A. J. A. M. Sips, and R. E. Geertsma, "Particle size-dependent organ distribution of gold nanoparticles after intravenous administration," Biomaterials, vol. 29, no. 12, pp. 1912-1919, Apr. 2008, doi: 10.1016/j.biomaterials.2007.12.037.

[9] B. D. Chithrani, A. A. Ghazani, and W. C. W. Chan, "Determining the Size and Shape Dependence of Gold Nanoparticle Uptake into Mammalian Cells," Nano Lett., vol. 6, no. 4, pp. 662-668, Apr. 2006, doi: 10.1021/n1052396o.

[10] E. A. Van Doren, P.-J. R. De Temmerman, M. A. D. Francisco, and J. Mast, "Determination of the volume-specific surface area by using transmission electron tomography for characterization and definition of nanomaterials," $J$ Nanobiotechnol, vol. 9, no. 1, p. 17, Dec. 2011, doi: 10.1186/1477-3155-9-17.

[11] L. Wang, X. Jiang, Y. Ji, R. Bai, Y. Zhao, X. Wu, and C. Chen, "Surface chemistry of gold nanorods: origin of cell membrane damage and cytotoxicity," Nanoscale, vol. 5, no. 18, p. 8384, 2013, doi: 10.1039/c3nr01626a.

[12] C. Uboldi, D. Bonacchi, G. Lorenzi, M.I. Hermanns, C. Pohl, G. Baldi, R. E. Unger, and C. J. Kirkpatrick, "Gold nanoparticles induce cytotoxicity in the alveolar type-II cell lines A549 and NCIH441," Part Fibre Toxicol, vol. 6, no. 1, p. 18, 2009, doi: 10.1186/1743-8977-6-18.

[13] H. C. Fischer and W. C. Chan, "Nanotoxicity: the growing need for in vivo study," Current Opinion in Biotechnology, vol. 18, no. 6, pp. 565-571, Dec. 2007, doi: 10.1016/j.copbio.2007.11.008.

[14] L. G. Griffith and M. A. Swartz, "Capturing complex 3D tissue physiology in vitro," Nat Rev Mol Cell Biol, vol. 7, no. 3, pp. 211-224, Mar. 2006, doi: 10.1038/nrm1858.

[15] J. J. Li, D. Hartono, C.-N. Ong, B.-H. Bay, and L.-Y. L. Yung, "Autophagy and oxidative stress associated with gold nanoparticles," Biomaterials, vol. 31, no. 23, pp. 5996-6003, Aug. 2010, doi: 10.1016/j.biomaterials.2010.04.014.

[16] T. Schneider, M. Westermann, and M. Glei, "In vitro uptake and toxicity studies of metal nanoparticles and metal oxide nanoparticles in human HT29 cells," Arch Toxicol, vol. 91, no. 11, pp. 3517-3527, Nov. 2017, doi: 10.1007/s00204017-1976-z.

[17] D. Mateo, P. Morales, A. Ávalos, and A. I. Haza, "Oxidative stress contributes to gold nanoparticle-induced cytotoxicity in human tumor cells," Toxicology Mechanisms and Methods, vol. 24, no. 3, pp. 161-172, Mar. 2014, doi: $10.3109 / 15376516.2013 .869783$.

[18] H. K. Patra, S. Banerjee, U. Chaudhuri, P. Lahiri, and A. Kr. Dasgupta, "Cell selective response to gold nanoparticles," Nanomedicine: Nanotechnology, Biology and Medicine, vol. 3, no. 2, pp. 111-119, Jun. 2007, doi: 10.1016/j.nano.2007.03.005.

[19] A. A. Sousa, S. A. Hassan, L. L. Knittel, A. Balbo, M. A. Aronova, P. H. Brown, P. Schuck, and R. D. Leapman, "Biointeractions of ultrasmall glutathione-coated gold nanoparticles: effect of small size variations," Nanoscale, vol. 8, no. 12, pp. 6577-6588, 2016, doi: 10.1039/C5NR07642K.

[20] J. J. Li, L. Zou, D. Hartono, C.-N. Ong, B.-H. Bay, and L.-Y. Lanry Yung, "Gold Nanoparticles Induce Oxidative Damage in Lung Fibroblasts In Vitro," Adv. Mater., vol. 20, no. 1, pp. 138-142, Jan. 2008, doi: 10.1002/adma.200701853.

[21] M. Auffan, J. Rose, T. Orsiere, M. De Meo, A. Thill, O. Zeyons, O. Proux, A. Masion, P. chaurand, O. Spalla, A. Botta, M. R. Wiesner, and J. Bottero, "CeO $\mathrm{Ce}_{2}$ nanoparticles induce DNA damage towards human dermal fibroblasts in vitro," Nanotoxicology, vol. 3, no. 2, pp. 161-171, Jan. 2009, doi: 10.1080/17435390902788086.

[22] I. Ojea-Jiménez and V. Puntes, "Instability of Cationic Gold Nanoparticle Bioconjugates: The Role of Citrate Ions," J. Am. Chem. Soc., vol. 131, no. 37, pp. 13320-13327, Sep. 2009, doi: 10.1021/ja902894s.

[23] N. J. Siddiqi, M. A. K. Abdelhalim, A. K. El-Ansary, A. S. Alhomida, and W. Y. Ong, "Identification of potential biomarkers of gold nanoparticle toxicity in rat brains," J Neuroinflammation, vol. 9, no. 1, p. 656, Dec. 2012, doi: 10.1186/1742-2094-9-123. 
[24] C. Lopez-Chaves, J. Soto-Alvaredo, M. Montes-Bayon, J. Bettmer, J. Llopis, and C. Sanchez-Gonzalez, "Gold nanoparticles: Distribution, bioaccumulation and toxicity. In vitro and in vivo studies," Nanomedicine: Nanotechnology, Biology and Medicine, vol. 14, no. 1, pp. 1-12, Jan. 2018, doi: 10.1016/j.nano.2017.08.011.

[25] S. K. Balasubramanian, J. Jittiwat, J. Manikandan, C.-N. Ong, L. E. Yu, and W.-Y. Ong, "Biodistribution of gold nanoparticles and gene expression changes in the liver and spleen after intravenous administration in rats," Biomaterials, vol. 31, no. 8, pp. 2034-2042, Mar. 2010, doi: 10.1016/j.biomaterials.2009.11.079.

[26] C. Lasagna-Reeves, D. Gonzalez-Romero, M.A. Barria, I. Olmedo, A. Clos, V.M. Sadagopa Ramanujam, A. Urayama, L. Vergara, M. J. Kogan, and C. Soto, "Bioaccumulation and toxicity of gold nanoparticles after repeated administration in mice," Biochemical and Biophysical Research Communications, vol. 393, no. 4, pp. 649-655, Mar. 2010, doi: 10.1016/j.bbrc.2010.02.046.

[27] J. Lipka, M. Semmler-Behnke, R. A. Sperling, A. Wenk, S. Takenaka, C. Schleh, T. Kissel, W. J. Parak, and W. G. Kreyling, "Biodistribution of PEG-modified gold nanoparticles following intratracheal instillation and intravenous injection," Biomaterials, vol. 31, no. 25, pp. 6574-6581, Sep. 2010, doi: 10.1016/j.biomaterials.2010.05.009.

[28] X. Li, Z. Hu, J. Ma, X. Wang, Y. Zhang, W. Wang, and Z. Yuan, "The systematic evaluation of size-dependent toxicity and multi-time biodistribution of gold nanoparticles," Colloids and Surfaces B: Biointerfaces, vol. 167, pp. 260-266, Jul. 2018, doi: 10.1016/j.colsurfb.2018.04.005.

[29] B. Yahyaei, M. Nouri, S. Bakherad, M. Hassani, and P. Pourali, "Effects of biologically produced gold nanoparticles: toxicity assessment in different rat organs after intraperitoneal injection," AMB Expr, vol. 9, no. 1, p. 38, Dec. 2019, doi: 10.1186/s13568-019-0762-0.

[30] E. C. Cho, J. Xie, P. A. Wurm, and Y. Xia, "Understanding the Role of Surface Charges in Cellular Adsorption versus Internalization by Selectively Removing Gold Nanoparticles on the Cell Surface with a I 2 /KI Etchant," Nano Lett., vol. 9, no. 3, pp. 1080-1084, Mar. 2009, doi: 10.1021/nl803487r.

[31] Y.-S. Chen, Y.-C. Hung, I. Liau, and G. S. Huang, "Assessment of the In Vivo Toxicity of Gold Nanoparticles," Nanoscale Res Lett, vol. 4, no. 8, p. 858, Aug. 2009, doi: 10.1007/s11671-009-9334-6.

[32] E. I. Hassanen, E. A. Morsy, A. M. Hussien, M. A. Ibrahim, and K. Y. Farroh, "The effect of different concentrations of gold nanoparticles on growth performance, toxicopathological and immunological parameters of broiler chickens," Bioscience Reports, vol. 40, no. 3, p. BSR20194296, Mar. 2020, doi: 10.1042/BSR20194296. 